\title{
Thermal Runaways in LHC Interconnections: Experiments
}

\author{
G. P. Willering, L. Bottura, P. Fessia, C. Scheuerlein, and A. P. Verweij
}

\begin{abstract}
The incident in the LHC in September 2008 occurred in an interconnection between two magnets of the $13 \mathrm{kA}$ dipole circuit. This event was traced to a defect in one of the soldered joints between two superconducting cables stabilized by a copper busbar. Further investigation revealed defective joints of other types. A combination of (1) a poor contact between the superconducting cable and the copper stabilizer and (2) an electrical discontinuity in the stabilizer at the level of the connection can lead to an unprotected quench of the busbar. Once the heating power in the unprotected superconducting cable exceeds the heat removal capacity a thermal run-away occurs, resulting in a fast melt-down of the non-stabilized cable. We have performed a thorough investigation of the conditions upon which a thermal run-away in the defect can occur. To this aim, we have prepared heavily instrumented samples with well-defined and controlled defects. In this paper we describe the experiment, and the analysis of the data, and we summarize the main results which are relevant to delimit the conditions of safe operation for the LHC. The results obtained, and specifically a controlled replica of the September 2008 incident demonstrate the necessity to re-work all main-circuit interconnects in the LHC in order to operate safely at the nominal energy.
\end{abstract}

Index Terms-Accelerators, copper stabilizer, LHC, thermal runaway.

\section{INTRODUCTION}

$\mathbf{T}$ HE incident in the LHC in September 2008 occurred in an interconnection between two magnets of the $13 \mathrm{kA}$ dipole circuit. It delayed the Large Hadron Collider (LHC) operation at CERN for more than one year. Detailed investigation revealed the signs of an interconnection with an excessively high joint resistance, estimated between 180 and $260 \mathrm{n} \Omega$ at a temperature of $1.9 \mathrm{~K}$ [1]. This resistance caused a heating of the interconnection, but remained stable during a 1 hour run at $7 \mathrm{kA}$. During the following run with a ramp rate of $10 \mathrm{~A} / \mathrm{s}$ the temperature exceeded the critical temperature $\left(T_{c}\right)$ of the superconducting cable and at $8.7 \mathrm{kA}$ a non-recoverable thermal runaway initiated.

Besides the high-resistance joint a propagating normal zone from a magnet or a wave of warm helium gas due to the quench of one of the magnets can be a cause for warming up the interconnection to above $T_{c}$, forcing the current through the copper stabilizer. The cross-section of the copper stabilizer is designed

Manuscript received August 02, 2010; accepted October 29, 2010. Date of publication December 23, 2010; date of current version May 27, 2011.

The authors are with the European Organization for Nuclear Research (CERN), CH-1211 Geneva 23, Switzerland (e-mail: Gerard. Willering@cern.ch).

Color versions of one or more of the figures in this paper are available online at http://ieeexplore.ieee.org.

Digital Object Identifier 10.1109/TASC.2010.2093114

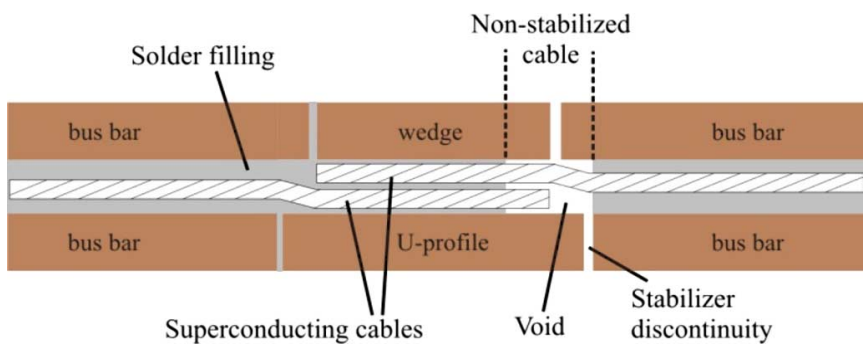

Fig. 1. Schematic drawing of an LHC splice with a single defect on the right hand side of the interconnection. The length of non-stabilized cable is the endangered part of the interconnection.

to maintain the current during the ramp-down of current without overheating the interconnection.

However, during the repairs of sector 3-4, multiple techniques (gamma ray imaging and DC electrical resistance measurements) revealed several defective interconnections with excess electrical resistance [2]. Ideally the copper stabilizers are filled completely with solder and a good electrical contact between the copper stabilizers is realized, see the left half of Fig. 1. On the right half of Fig. 1 one of the common defects is shown, with two coexisting features:

1) A lack of contact between the superconducting cable and the stabilizer due to missing solder.

2) An electrical discontinuity between the stabilizers.

A defect is characterized by the length of non-stabilized cable $\left(L_{n s c}\right)$ and the residual resistance ratio (RRR) of the superconducting cable. $L_{n s c}$, also called "defect length" is typically in the order of 15 to $50 \mathrm{~mm}$, with RRR roughly between 100 and 200 . With $L_{n s c}$ and RRR the defect resistance at low temperature and room temperature is defined.

Thorough investigation by computer modeling led to a risk analysis and a decision for the operation [3] and for the LHC repair schedule [4]. These decisions are important and require a well founded experimental validation. The found defects do not allow to safely operate the main busbars at their nominal design current of $13 \mathrm{kA}$ when the exponential current decay with a $\tau$ of 20 and 100 s for the Main Quadrupole (MQ) and Main Dipole (MB) circuits, respectively.

Here we summarize the experimental research on a series of MQ-circuit interconnection samples to investigate the influence of multiple interconnection characteristics (RRR, $L_{n s c}$, cooling, etc.) and to provide input for the validation of the calculation model. In Section II the details of the experiments are discussed. Thermal runaway measurements are presented in Section III, followed by a controlled burn out of one interconnection in Section IV. In Section V the measurements on shunted interconnection samples are discussed. 

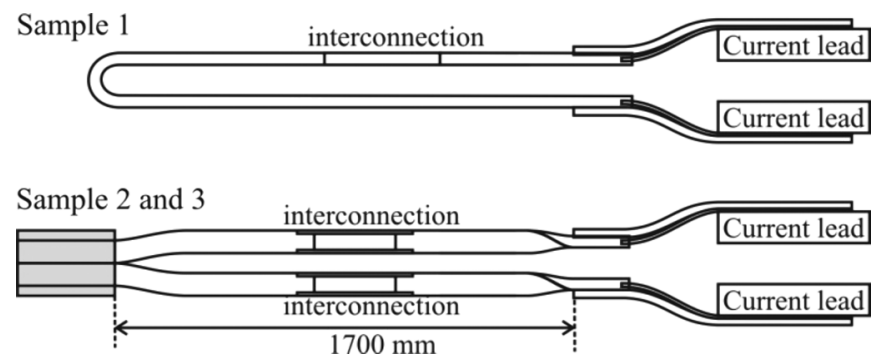

Fig. 2. Overview of the single interconnection in sample 1 and the two interconnections in sample 2, 3 and 4.

TABLE I

CHARACTERISTICS OF THE SAMPLES

\begin{tabular}{llllll}
\hline \hline Sample & $\begin{array}{l}L_{n s c} \\
(\mathrm{~mm})\end{array}$ & $\begin{array}{l}\text { Radd } \\
(\mathrm{T}=300 \mathrm{~K}) \\
(\mu \Omega)\end{array}$ & $\begin{array}{l}\text { Radd } \\
(\mathrm{T}=10 \mathrm{~K}) \\
(\mu \Omega)\end{array}$ & $\begin{array}{l}\text { RRR } \\
\text { Cable }\end{array}$ & $\begin{array}{l}\text { RRR } \\
\text { Busbar }\end{array}$ \\
\hline 1 & 47 & 63 & 0.37 & 170 & $\sim 300$ \\
2A & $35+27$ & $43+32$ & $0.43+0.24$ & $100-130$ & $\sim 270$ \\
2B & 35 & 42 & 0.26 & $\sim 170$ & $\sim 290$ \\
3A & $39+30$ & $51+39$ & $0.31+0.28$ & $140-170$ & $\sim 190$ \\
3B & 21 & 27 & 0.22 & 120 & $\sim 160$ \\
4A & $38+36$ & $50+48$ & - & - & $\sim 270$ \\
4B & 25 & 33 & - & - & $\sim 290$ \\
\hline \hline
\end{tabular}

\section{SAMPLE DESCRIPTION}

In total seven interconnection samples with different defects have been prepared and adapted to fit in the test station FRESCA. For sample 1 the rectangular shaped busbar (10 by $20 \mathrm{~mm}$ ) is bent into a $\mathrm{U}$-shape to complete the electrical circuit and incorporates one interconnection, see Fig. 2. The busbars face each other with the broad side. For sample 2, 3 and 4, the busbars are positioned in a similar configuration as in the LHC, facing each other with the small side. A soldered joint completes the electrical circuit. To measure the two interconnections in sample 2, 3 and 4 separately a well cooled copper block is added to the soldered joint, which prevents the normal zone to propagate from one side to the other side during the test.

The 1.7 meter long samples (with $0.2 \mathrm{~m}$ long interconnection regions) are submerged in liquid helium at $4.3 \mathrm{~K}$ or in superfluid helium at $1.9 \mathrm{~K}$. The tested interconnections are of the MQ type, with a copper cross-section of $148 \mathrm{~mm}^{2}$, which is about 12 times more than the cross-section of the superconducting cable of $12.6 \mathrm{~mm}^{2}$ [5].

In Table I the characteristics of the defective samples have been listed. Samples 2A, 3A and 4A incorporate a double defect with a non-stabilized cable on both sides of the splice, while samples 2B, 3B and 4B have a single defect. Samples 4A and 4B both incorporate a copper shunt and are discussed in Section V.

The sample, and specifically the interconnection region, is instrumented by:

- voltage taps soldered on top of the sample,

- thermo-couples placed in $0.5 \mathrm{~mm}$ diameter holes inside the busbar or U-profile,

- thermofoil heaters on the bottom side of the busbar and U-profile to initiate a normal conducting zone.
TABLE II

DIFFERENCE BETWEEN LHC AND TEST CONDITIONS

\begin{tabular}{lll}
\hline \hline Symbol & LHC conditions & FRESCA setup \\
\hline Current profile & $\begin{array}{l}\text { Exponential decay } \\
\tau=100 \mathrm{~s} \text { (MB-circuit) } \\
\tau=10 \text { to } 20 \mathrm{~s} \text { (MQ-circuit) }\end{array}$ & $\begin{array}{l}\text { Constant current } \\
(\tau=\infty)\end{array}$ \\
$\begin{array}{l}\text { Effective cooled } \\
\text { busbar surface }\end{array}$ & $\sim 90 \%$ & $\sim 80 \%$ \\
Busbar length & $>5 \mathrm{~m}$ & $<0.8 \mathrm{~m}$ \\
Helium cooling & Liquid He or gaseous He & Only liquid He \\
RRR busbar & 100 (worst case) & $160-310$ \\
RRR cable & 80 (worst case) & $100-170$ \\
\hline \hline
\end{tabular}

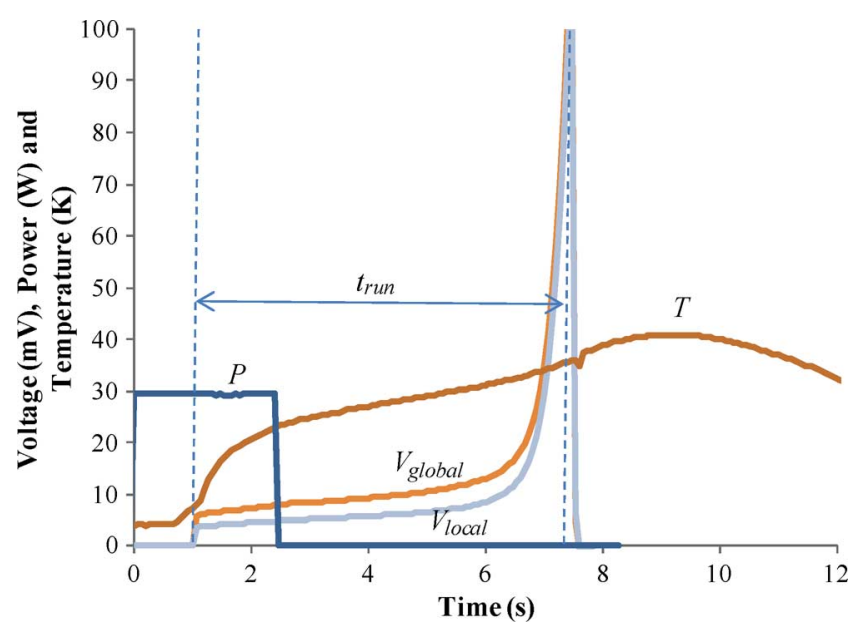

Fig. 3. Typical measurement sequence: A heat pulse $(P)$ makes temperature $(T)$ to rise, followed by a normal zone in the superconductor with a step in the voltage on the full sample length $\left(V_{\text {global }}\right)$ and on the interconnection region $\left(V_{\text {local }}\right)$. Sample $2 \mathrm{~A}$ is shown with $I=7 \mathrm{kA}, T=1.9 \mathrm{~K}, B=0 \mathrm{~T}$ and $t_{\text {run }}=6.4 \mathrm{~s}$.

The sample conditions during test are different from LHC operation conditions. Therefore direct comparison of results with operation conditions is not possible. Table II indicates the main differences between LHC and test conditions. Due to the constant current the sample is less stable, while the short busbar length ( 0.8 meter to a well stabilized part on each side of the interconnection) improves stability due to more cooling through the busbar to the cold end. The busbar is insulated from the helium bath by interlaced layers of Kapton tape in a standard LHC insulation scheme. However at some spots clamping by G10 pieces reduce the effectively cooled busbar surface. The sample does not meet the worst case conditions in the LHC, since a higher RRR in the sample improves stability.

A typical measurement sequence starts with ramping up the current to a constant level followed by a short heat pulse of about 2 seconds. The heater power is tuned to start a normal zone after 1 second. In case of a thermal runaway the voltage on the sample, and notably the voltage on the interconnection, increases and a runaway of the voltage appears, see Fig. 3. At a threshold of $100 \mathrm{mV}$ the current supply is switched off to prevent the sample from burn-through. The temperature of the busbar at $15 \mathrm{~mm}$ from the hot spot rises even after the heating stops to about $40 \mathrm{~K}$, indicating a higher hot spot temperature 


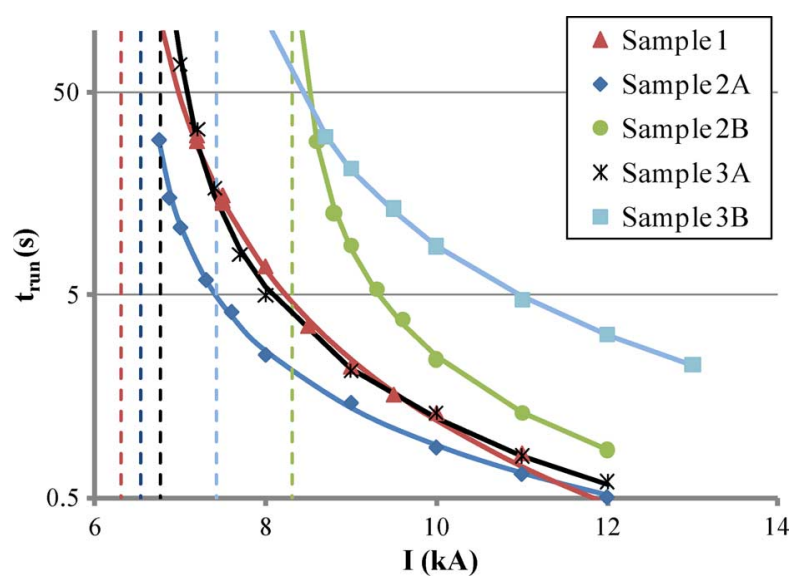

Fig. 4. Thermal runaway time for the first five samples as a function of current at a temperature of $1.9 \mathrm{~K}$ in self-field. The dashed lines indicate $\mathrm{I}_{\mathrm{ss}}$, as obtained by curve fitting.

than the measured temperature. Estimations with the temperature-resistance of $\mathrm{Cu}$ relation show $T_{\text {hotspot }}<100 \mathrm{~K}$.

One of the major characteristics extracted from the measurements is the thermal runaway time $t_{\text {run }}$, the time between the start of the normal zone and the current cut-off. The shunted samples show a much slower thermal runaway, therefore $t_{\text {run }}$ is characterized at an estimated shunt temperature of $300 \mathrm{~K}$, see Section V.

\section{THERMAL RUNAWAYS}

In total about 200 thermal runaways have been created during measurements in FRESCA in varying cooling conditions $(4.3 \mathrm{~K}$ and $1.9 \mathrm{~K}$ ) and varying magnetic fields (from self-field to $9 \mathrm{~T}$ ). By varying the transversely applied field, a higher defect and busbar resistance of a factor of more than 2 can be simulated by the magnetoresistive effects of copper, thus increasing the measurement range.

Fig. 4 shows the measured $t_{\text {run }}$ as function of current for the five non-shunted samples. An empirical fit by (1) applies well to the curves:

$$
t_{\text {run }}=a\left(I-I_{s s}\right)^{-b}
$$

with $a\left[A^{b} / s\right]$ and $b[-]$ are fit parameters. $I_{s s}$ indicates the steady state current when the cooling is in equilibrium with the heating, hence the normal zone will neither propagate nor recover.

In case of a magnet quench or other fast abort events, the current in the LHC MQ-circuits decays exponentially with $\tau=$ $20 \mathrm{~s}$. The MIITs deposited in the circuit are similar as for a constant current for $10 \mathrm{~s}$, therefore the characteristic current is obtained from the curves in Fig. 4 at $t_{\text {run }}=10 \mathrm{~s}$. In Fig. 5 the characteristic current is shown as a function of the additional resistance of the sample. The additional heating power $\left(I^{2} R_{\text {add }}\right)$ that is needed to cause a thermal runaway after 10 seconds is between 16 and $27 \mathrm{~W}$ for all the different samples, see Fig. 5 . Although the correlation is empirically found, it is indicative how much additional resistive heating power can be allowed in a $10 \mathrm{~s}$ time scale to initiate the thermal runaway.

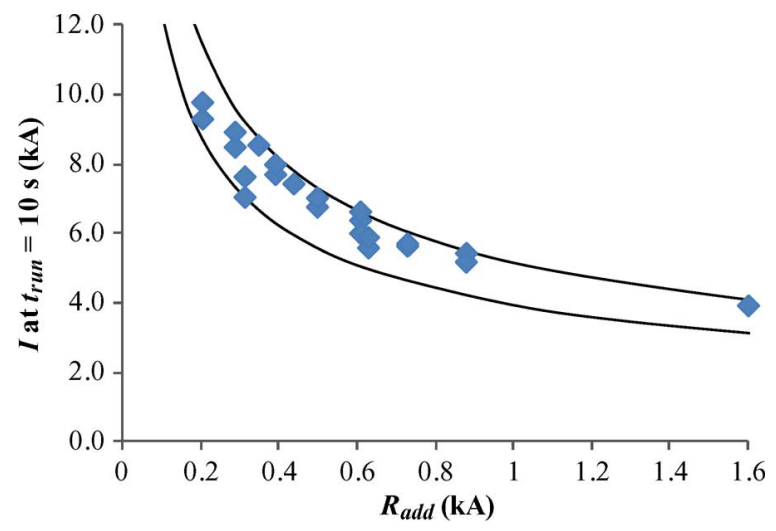

Fig. 5. The current which gives a runaway time of 10 seconds as function of the additional resistance of the samples at $10 \mathrm{~K}$. The lines are constant heating power fits with $I^{2} R=16$ and $27 \mathrm{~W}$.

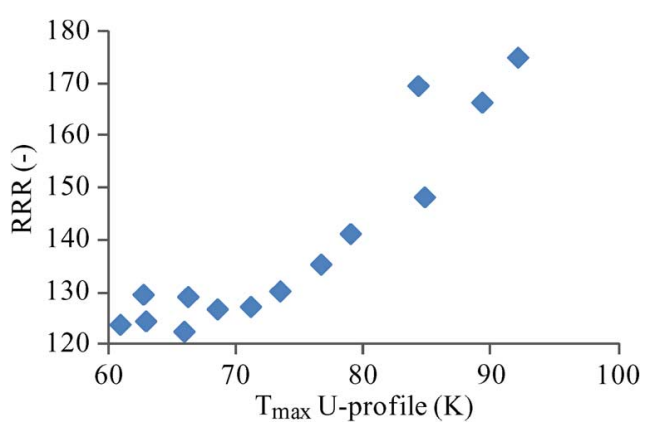

Fig. 6. Measured average RRR of the non-stabilized cable versus the measured maximum temperature of the busbar at $15 \mathrm{~mm}$ from the hot spot.

\section{NON-PROTECTED THERMAL RUNAWAY}

After finishing the measurements of sample $3 \mathrm{~A}$ we increased the protection threshold from 100 to $500 \mathrm{mV}$ in 14 steps. The maximum temperature measured with a thermocouple inside the busbar at $15 \mathrm{~mm}$ from the hot spot remained below $100 \mathrm{~K}$. Resistance measurements of the non-stabilized cable between thermal runaways show a reduction of the resistance by $30 \%$, see Fig. 6. Since the copper is annealed during the short heat pulses local temperatures must have been above $500 \mathrm{~K}$.

At the final step the cable burned through, hence the hot spot temperature exceeded the copper melting temperature of 1360 K. On the photograph and the gamma-ray image shown in Fig. 7, the gap of 1.5 to $3 \mathrm{~mm}$ wide across the full cable width is visible. The experiment shows the capability of reproducing the September 2008 incident, although having slightly different experimental conditions. During this measurement about $1.6 \mathrm{~kJ}$ was deposited in the sample while the LHC main circuits hold a more destructive stored magnetic energy of $20 \mathrm{MJ}$ (MQ-circuit) to $1.2 \mathrm{GJ}$ (MB-circuit).

\section{SHUNTED INTERCONNECTION}

To consolidate the main splices for nominal operation of the LHC at $7 \mathrm{TeV}$ it has been proposed to add shunts with a $3 \mathrm{~mm} \times$ $15 \mathrm{~mm}$ cross-section to all interconnections [6]. A first indicative validation experiment of the stability of a shunted cable has been performed in FRESCA on samples 4A and 4B. To prepare a well-defined sample with a relatively long non-soldered shunt 


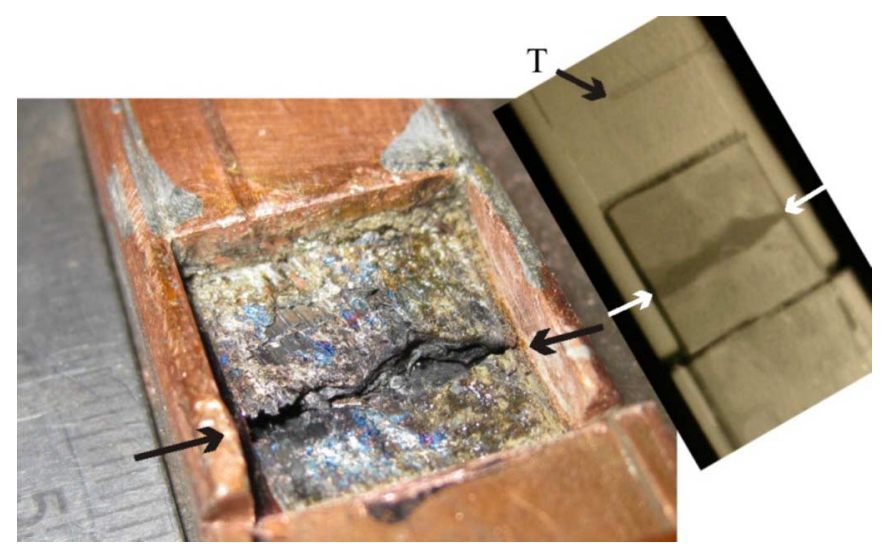

Fig. 7. Photograph of the burned-through sample after removal of the top layer of the stabilizer, with the gap in between the arrows. The inset on the right shows a gamma-ray image with a top-view, the dark area across the width indicates the hole in the cable. $T$ indicates the thermocouple position inside the busbar.

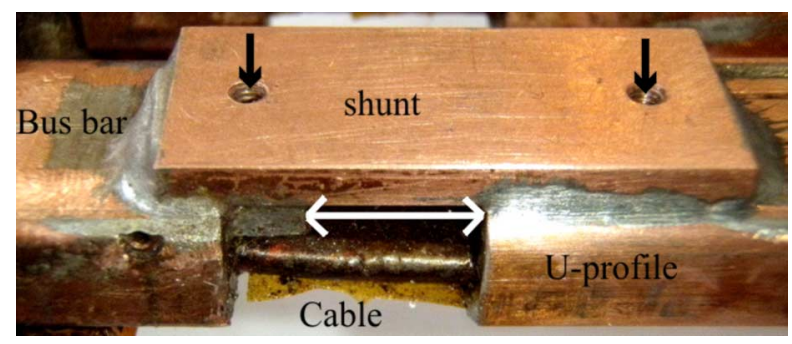

Fig. 8. One of the shunts soldered on sample 4. The white arrow indicates the non-soldered shunt length. A part of the U-profile is removed to avoid contact with the busbar, therefore the non-stabilized part of the cable is also visible. The screw holes on top of the shunt, indicated by black arrows, are used to fix the voltage taps.

TABLE III

CHARACTERISTICS OF THE SHunTEd SAMPLES

\begin{tabular}{llll}
\hline \hline Sample & $\begin{array}{l}\mathrm{L}_{\mathrm{NSS}} \\
(\mathrm{mm})\end{array}$ & $\begin{array}{l}\text { Shunt } \\
\text { Thickness } \\
(\mathrm{mm})\end{array}$ & $\begin{array}{l}\mathrm{R}_{\text {add }} \\
(\mathrm{T}=300 \mathrm{~K}) \\
(\mu \Omega)\end{array}$ \\
\hline $4 \mathrm{~A}-3 \mathrm{~mm}$ & $7 \& 5$ & 3 & $3.9 \& 3.1$ \\
$4 \mathrm{~A}-1.5 \mathrm{~mm}$ & $7 \& 5$ & 1.5 & $6.4 \& 5.4$ \\
$4 \mathrm{~B}-3 \mathrm{~mm}$ & $11 \& 0$ & 3 & $2.8 \&-0.5$ \\
$4 \mathrm{~B}-1.5 \mathrm{~mm}$ & $11 \& 0$ & 1.5 & $5.1 \&-0.1$ \\
\hline \hline
\end{tabular}

length $L_{n s s}$ and to avoid the leakage of $\mathrm{SnPb}$ solder making contact between the busbar and the U-profile, a part of the U-profile is removed and filled with non-conductive G10 pieces, see Fig. 8. During the consolidation of the LHC, foreseen in 2012, an $L_{n s s}$ of $8 \mathrm{~mm}$ has been guaranteed. For the test a more conservative value is taken of 5 to $11 \mathrm{~mm}$.

To provide more measurement validation data the shunt is reduced to $1.5 \mathrm{~mm}$ thickness after the first test. In Table III the additional resistance of the shunted sample is listed as a function of the shunt thickness.

Compared to the non-shunted condition (see Table I), a resistance reduction by a factor of 11 to 15 is obtained with a $3 \mathrm{~mm}$ shunt and 6 to 9 for a $1.5 \mathrm{~mm}$ shunt.

A clear improvement of the thermal runaway time for a constant current condition is revealed by the measurements shown in Fig. 9.

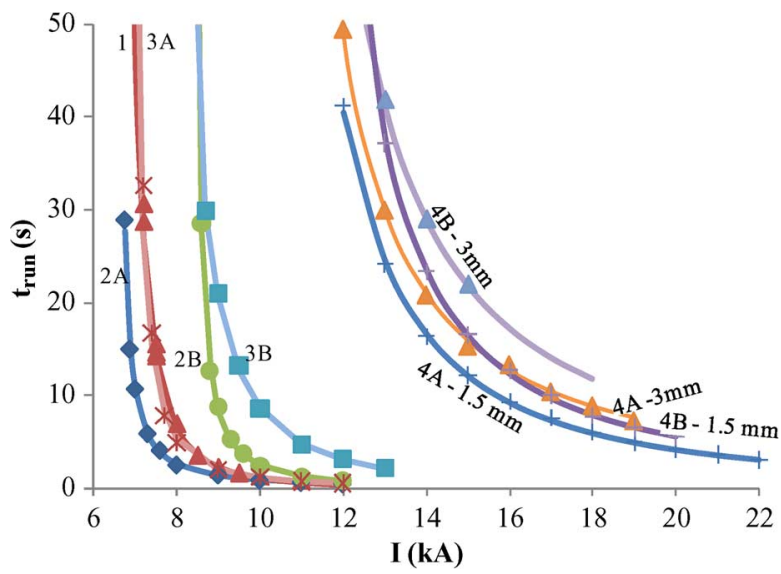

Fig. 9. Runaway time as a function of current for the non-shunted samples 1 to $3 \mathrm{~B}$ and the shunted samples $4 \mathrm{~A}$ and $4 \mathrm{~B} .1 .5$ and $3 \mathrm{~mm}$ indicate the shunt thickness.

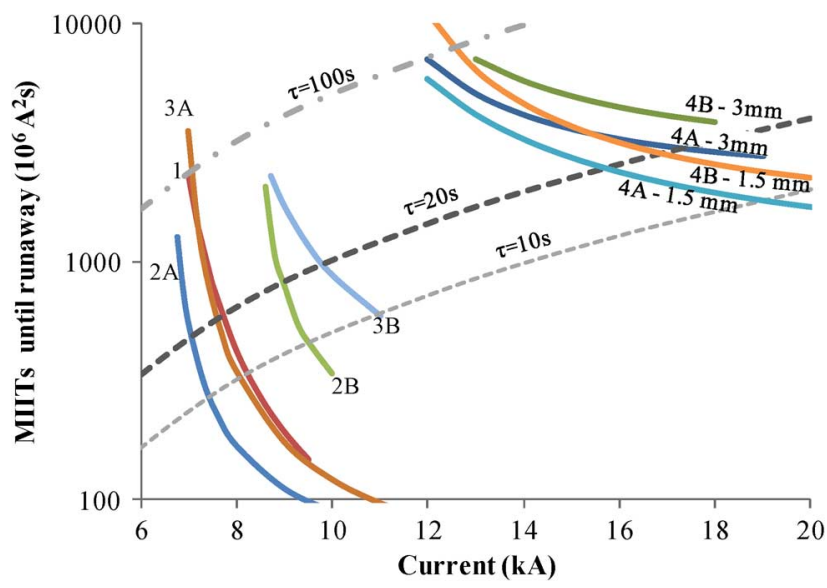

Fig. 10. The measured MIITs until the thermal runaway starts as a function of current. Samples 1 to $3 \mathrm{~B}$ are not shunted, while sample 4A and 4B exhibit 1.5 or $3 \mathrm{~mm}$ thick shunts. The dashed lines indicate the MIITs in the LHC circuits with a $\tau$ of 10,20 and $100 \mathrm{~s}$.

The short sample length gives a more stable boundary condition and the constant current makes it less stable. Therefore the test situation in not directly applicable to the LHC operation conditions. However, to provide an indication of the improvement due to the shunt, we characterized the curves with the MIITs which are induced in the sample until $t_{\text {run }}$. Fig. 10 shows a comparison between the shunted and non-shunted samples. The current MQ-circuit time constant for the LHC is $10 \mathrm{~s}$, whereas in Fig. 10 a safe current of about $7 \mathrm{kA}$ is suggested for all samples.

In the test situation, the shunted sample can hold the $13 \mathrm{kA}$ nominal current for more than 20 seconds. Nominal operation conditions with $\tau=20 \mathrm{~s}$ are foreseen after shunting all the interconnections. In the test situation the shunted samples, which do not represent the worst case scenario and are better cooled than the LHC condition, can take MIITs as in LHC operation conditions with $13 \mathrm{kA}$ [7].

The difference between 1.5 and $3 \mathrm{~mm}$ shunt thickness is relatively small for sample 4A and 4B, see Figs. 9 and 10. The voltages measured on one shunt show a very similar voltage buildup for both cases, indicating a similar increase of temperature for 


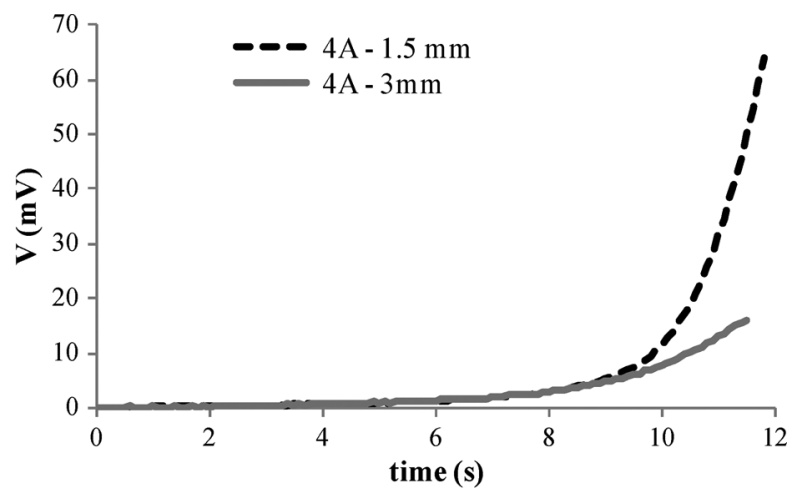

Fig. 11. Measured voltage build-up in time for the sample $4 \mathrm{~A}$ at $15 \mathrm{kA}$ with a 1.5 and $3 \mathrm{~mm}$ thick shunt. For voltagetap positions, see Fig. 8.

both shunt thicknesses, see Fig. 11. However, the $1.5 \mathrm{~mm}$ shunt starts to heat up much quicker from about 9 seconds than the 3 $\mathrm{mm}$ shunt and will burn through sooner.

One explanation for the relatively small difference between both shunts comes from resistance measurements, showing a relatively large resistance for the $\mathrm{SnPb}$ solder compared to the shunt resistance at $10 \mathrm{~K}$. However, the measured RRR for the soldered $\mathrm{SnPb}$ is low (well below 50) and the RRR of the annealed copper shunts is high (about 200). Therefore in the early stage of the thermal run away, most of the power is generated in the soldered area, which is similar for the 1.5 and the $3 \mathrm{~mm}$ shunt. At higher temperatures the influence of the solder becomes much less important, since it is in contact with the bus and therefore well-cooled. Therefore the difference between the 1.5 and $3 \mathrm{~mm}$ thick shunts is relatively larger at higher temperatures.

The dipole busbars with a larger copper cross section (280 $\mathrm{mm}^{2}$ ) are designed to carry decaying current with $\tau=100 \mathrm{~s}$ and an initial current of $13 \mathrm{kA}$. They are more critical due to the long time constant. The quadrupole busbar samples with $3 \mathrm{~mm}$ shunt support similar MIITs as $12 \mathrm{kA}$ operation with $\tau=100 \mathrm{~s}$, but without taking the worst case conditions into account.

\section{CONCLUSIONS}

Thermal run away measurements showed the imminent danger of the defective interconnections for nominal LHC operation at $7 \mathrm{TeV}$. Although the measurement conditions differ from the LHC conditions, they provide a good indication of allowable current and, moreover, they provided the necessary input for the simulation model. The indicative measurements on the shunted interconnections clearly show improved current carrying capabilities.

To validate the final design of the shunt an experiment is set up with conditions very close to LHC conditions. Two special short straight section magnets (SSS) of 7 and 8 meter length will be placed in series and the main dipole busbars going through the SSS magnets are connected providing a realistic test for the most critical circuit.

\section{ACKNOWLEDGMENT}

The authors are grateful to the input of many in discussing the measurements and for technical support. Many thanks go to Khalid Chaouki, Jean-Michel Dalin, Simon Heck, Sandrine le Naour, Giuseppe Peiro, Thierry Regnalia, Rosario Principe, Stephan Triquet, David Richter, the task force LHC splices consolidation and other participating members of the TE/MSC group.

\section{REFERENCES}

[1] P. Lebrun et al., Report of the Task Force on the Incident of 19 September 2008 at the LHC CERN LHC Project Report 1168, March 31, 2009.

[2] C. Scheuerlein et al., "Production and quality assurance of the interconnection splices of the main busbars during the LHC 2008-2009 shutdown," in ASC 2010, submitted for publication.

[3] A. P. Verweij, "Thermal runaway of the $13 \mathrm{kA}$ busbar joints in the LHC," IEEE Trans. Appl. SC, vol. 20, no. 3, pp. 2155-2159, 2010

[4] A. P. Verweij, "Minimum requirements for the $13 \mathrm{kA}$ splices for $7 \mathrm{TeV}$ operation," in Proceedings LHC Performance Workshop, Chamonix, 2010.

[5] L. Belova, M. Genet, J. Perinet-Marquet, P. Ivanov, and C. Urpin, "Design and manufacture of the superconducting busbars for the LHC main magnets," IEEE Trans. Appl. Sc., vol. 12, pp. 1305-1309, 2002.

[6] F. Bertinelli et al., "Towards a consolidation of LHC superconducting splices for $7 \mathrm{TeV}$ operation," in IPAC 2010, Kyoto, Japan, May 2010.

[7] CERN, LHC Design Report Geneva, Switzerland, EDMS 445830 and 445839, 2004. 